\title{
Association of CA27.29 and Circulating Tumor Cells Before and at Different Times After Adjuvant Chemotherapy in Patients with Early-stage Breast Cancer - The SUCCESS Trial
}

\author{
PHILIP HEPP ${ }^{1}$, ULRICH ANDERGASSEN ${ }^{2}$, BERNADETTE JÄGER $^{3}$, ELISABETH TRAPP $^{2}$, \\ MARIANNA ALUNNI-FABBRONI ${ }^{2}$, THOMAS W.P. FRIEDL ${ }^{4}$, NADESCHDA HECKER ${ }^{5}$, RALF LORENZ ${ }^{5}$, \\ PETER FASCHING $^{6}$, ANDREAS SCHNEEWEISS ${ }^{7}$, TANJA FEHM ${ }^{3}$, WOLFGANG JANNI ${ }^{4}$ and BRIGITTE RACK ${ }^{2}$ \\ ${ }^{1}$ Department of Gynecology and Obstetrics, HELIOS University Hospital Wuppertal, \\ University Witten/Herdecke, Wuppertal, Germany; \\ ${ }^{2}$ Department of Gynecology and Obstetrics, University Hospital Ludwig-Maximilian University, Munich, Germany; \\ ${ }^{3}$ Department of Gynecology and Obstetrics, University Hospital Heinrich-Heine University, Düsseldorf, Germany; \\ ${ }^{4}$ Department of Gynecology and Obstetrics, University Hospital Ulm, Ulm, Germany; \\ ${ }^{5}$ Joint practice for Gynecology and Obstetrics, Braunschweig, Germany; \\ ${ }^{6}$ Department of Gynecology and Obstetrics, \\ University Hospital Friedrich-Alexander University, Erlangen, Germany; \\ ${ }^{7}$ National Center for Tumor Diseases, University Hospital Heidelberg, Heidelberg, Germany: \\ SUCCESS Studiengruppe
}

\begin{abstract}
Background: Evidence for the prognostic value of circulating tumor cells (CTCs) in early-stage breast cancer is swiftly increasing. An alternative approach for identifying patients at risk for recurrence is based on the detection of the mucin-1 (MUC1)-based tumor marker CA27.29. Here we report the association of these two prognostic markers before and immediately after chemotherapy (CHT), as well as after 2 and 5 years of follow-up. Patients and Methods: The SUCCESS trial compared fluorouracil, epirubicin and cyclophosphamide followed by docetaxel vs. FEC followed by docetaxel plus gemcitabine, and 2 vs. 5 years of treatment with zoledronic acid in 3,754 patients with node-positive or high-risk nodenegative early-stage breast cancer. CA27.29 was measured with the ST AIA-PACK CA27.29 reagent (Tosoh Bioscience, Belgium). The cutoff for CA27.29 positivity was $>31 \mathrm{U} / \mathrm{ml}$. CTCs were assessed with the CellSearch System (Veridex, USA). The cutoff for CTC positivity was $\geq 1$ CTC/15 ml whole blood. The relationship between CTC positivity and CA27.29 positivity was assessed based on Chi-square
\end{abstract}

Correspondence to: Dr. Philip Hepp, Landesfrauenklinik, Heusnerstr. 40, 42283 Wuppertal, Germany. Tel: +49 02028961401, e-mail: philip.hepp@helios-kliniken.de

Key Words: Tumor marker, minimal residual disease, adjuvant, breast cancer. statistics and Cramer's $V$, which varies from 0 (no association between the variables) to 1 (complete association). Samples for CA27.29 and CTC determinations during follow-up were only drawn from patients that had no relapse. Results: Both CA27.29 and CTC data were available for 1,981, 1,602, 1,159 and 707 patients before, immediately after and at 2 and 5 years after CHT, respectively. Positivity rates for CTC were $21.3 \%, 22.8 \%, 18.6 \%$ and $8.5 \%$, respectively. CA27.29 was positive in $7.9 \%, 21.0 \%, 2.8 \%$ and $7.5 \%$, respectively. Positivity for both CA27.29 and CTC was found in 2.4\%, 4.2\%, $0.7 \%$ and $1.8 \%$ of patients, respectively. The association between CA27.29 and CTC was significant but weak before CHT ( $p=0.0015$; Cramer's $V=0.063)$ and 5 years after CHT $(p<0.001$; Cramer's $V=0.164)$, and not significant immediately after $C H T$ $(p=0.162$; Cramer's $V=0.035)$ and 2 years after $(p=0.349$; Cramer's V=0.028). Conclusion: We showed that CTC and CA27.29 positivity were significantly, but only weakly associated before CHT and 5 years after CHT, while no significant association was found immediately or 2 years after CHT during the course of early-stage breast cancer. It, therefore, seems reasonable to further evaluate the prognostic value of CTCs and CA27.29 as a combined prognostic test of two potentially independent markers that might provide complementary prognostic information.

Breast cancer is still not only the most common cancer, but also the leading cause of cancer-related death amongst 
women (1). Of all women treated for early-stage breast cancer, about $30 \%$ will experience relapse within 10 years. There are several known markers such as tumor size or nodal status that help estimate prognosis at the time of surgery. Yet, there is no single marker to reliably identify those women at highest risk of relapse.

According to the current model residual tumor cells (after surgery and primary systemic therapy) are the cause of later relapse (2). This minimal residual disease has received increasing attention as a prognostic marker, in both the metastatic and adjuvant settings. The prognostic impact of disseminated tumor cells in the bone marrow of patients with early-stage breast cancer, as assessed at the time of primary diagnosis has been shown convincingly in a meta-analysis of 4,703 women (3). Recently, an independent prognostic value has also been demonstrated for circulating tumor cells (CTCs) in the blood of patients with early breast cancer $(4,5)$.

Another feasible approach to identify patients at high risk of relapse is the detection of cancer cell-specific products. Mucin 1 (MUC1)-derived tumor markers such as CA15-3 and CA27.29 have been shown to be of prognostic value in metastatic breast cancer and are routinely used in the management of patients at this stage (6). Furthermore, there is evidence for their prognostic significance in early-stage breast cancer and in the follow-up of breast cancer survivors (7-10).

The presence of CTCs reflects early dissemination of tumor cells into the bloodstream (2), whereas CA27.29 reflects the amount of MUC1, which correlates closely with tumor burden $(11,12)$. Therefore it seems possible that both markers provide independent prognostic information and that the combination of both CTCs and CA 27-29 may yield better prognostic information than each of the two markers alone.

This study examines the prevalence and association of CTCs and CA27.29 in patients with early-stage breast cancer during treatment and follow-up. A strong association of CTCs and CA27.29 would suggest that the two markers are unlikely to provide independent prognostic information. In contrast, a weak association or no association might indicate the potential of the two markers to provide valuable independent and complementary prognostic information.

\section{Patients and Methods}

The German multicenter SUCCESS A study was conducted in 251 study centers all participating in the prospectively planned translational research sub-protocol. CTC and CA27.29 analysis, as well as the time points of blood sampling and the methodology described below were prospectively planned as exploratory analyses within the clinical study protocol. The study was approved by all responsible Ethical Boards and is in line with the Declaration of Helsinki and is registered with the European Union Drug Regulating Authorities Clinical Trials Register (number 2005-000490-21).

Primary surgery consisted of either breast conservation or mastectomy leading to R0 resection in all cases. Sentinel node dissection was performed in all $\mathrm{cN} 0$ patients, followed by complete axillary node dissection in cases with positive sentinel nodes. Patients with $\mathrm{cN}+$ disease primarily underwent axillary-node dissection. Radiotherapy was performed according to national guidelines, and was used in all cases that received breast-conserving treatment.

Eligible patients were defined as women with histologically confirmed invasive breast cancer (stages pT1-4, pN0-3, M0) whose tumors had been completely removed and who had an indication for adjuvant chemotherapy (CHT) (nodal positive or high risk nodal negative, i.e. $\mathrm{pT} \geq 2$, tumor grade 3 , age younger than 35 years or negative hormone receptor status). The SUCCESS A study compared the disease-free survival of randomized patients treated with three cycles of epirubicin $\left(100 \mathrm{mg} / \mathrm{m}^{2}\right)$ with fluorouracil $\left(500 \mathrm{mg} / \mathrm{m}^{2}\right)$ and cyclophosphamide $\left(500 \mathrm{mg} / \mathrm{m}^{2}\right)$ (FEC) every three weeks $(q 3 w)$ followed by three cycles of docetaxel $\left(100 \mathrm{mg} / \mathrm{mg}^{2}\right) q 3 w$ versus three cycles of FEC $q 3 w$ followed by three cycles of gemcitabine $\left(1,000 \mathrm{mg} / \mathrm{m}^{2}\right.$ days 1,8$)$ with docetaxel $\left(75 \mathrm{mg} / \mathrm{m}^{2}\right) q 3 w$. After completion of CHT, patients were further randomized to receive either 2 (every 3 months for 24 months) or 5 years of zoledronate (every 3 months for 24 months followed by every 6 months for 36 months). Women with hormone receptor-positive disease received endocrine treatment. Pre-menopausal women received tamoxifen alone or in combination with goserelin for two years if they were younger than 40 years of age or became pre-menopausal within 6 months of CHT. Postmenopausal patients were treated with tamoxifen for 2 years, followed by anastrozole for 3 years.

Blood was drawn into three CellSave preservative tubes $(30 \mathrm{ml}$ in total) containing a cell-stabilizing agent and one standard serum tube $(7.5 \mathrm{ml})$. It was shipped at room temperature to the central laboratory at the Women's Hospital of the University of Munich, Germany, and was analyzed within 72 hours after drawing.

For CTC analysis, $30 \mathrm{ml}$ of blood was pooled and reduced to 7.5 $\mathrm{ml}$ by centrifugation for 10 minutes at $800 \times \mathrm{g}$. Plasma was removed and dilution buffer added, and this mixture was gently overlaid over $6 \mathrm{ml}$ of Histopaque using a syringe with valve and tubing. After centrifugation for 10 minutes at $400 \times g, 7.5 \mathrm{ml}$ of this sample containing the buffy coat was subsequently processed with the CellSearch System (Janssen Diagnostics, Raritan, NJ, USA) as described elsewhere (13). The cell images created by the semiautomated CellTracks Analyzer were classified by two independent observers; a sample was considered CTC-positive if at least one CTC was found.

CA27.29 was measured by the ST AIA-Pack 27.29 reagent using the AIA-600II Analyzer (Tosoh Bioscience, Tessenderlo, Belgium) according to the manufacturer's instructions. The ST AIA-Pack 27.29 assay is an automated monoclonal fluorometric assay directed against MUC1 antigen. Serum (150 $\mu \mathrm{l})$ was diluted by 1:20 and the monoclonal antibody bound to magnetic beads was then added. The beads were washed and incubated at $37^{\circ} \mathrm{C}$ with the fluorogenic substrate 4-methylumbelliferyl phosphate. Following the manufacturer's recommendations, assay results above $31 \mathrm{U} / \mathrm{ml}$ were regarded as positive.

Samples for CA27.29 and CTC determinations were taken before chemotherapy, immediately after CHT, and after 2 and 5 years of follow-up. However, blood sampling for CA27.29 and CTC measurements was restricted to patients that had not experienced relapse at the time.

Statistics. Statistical analysis was conducted in accordance with the main study protocol. Categorical variables were described 
Table I. Association of circulating tumor cell status (positive if $\geq 1$ CTC/15 ml blood) and CA27.29 status (positive if $>31 \mathrm{U} / \mathrm{ml}$; negative if $\leq 31$ $U / \mathrm{ml}$ ) with patient and tumor characteristics at baseline.

\begin{tabular}{|c|c|c|c|c|c|c|}
\hline Variable & $\begin{array}{l}\text { CTC-positive, } \\
\mathrm{n}(\%)(\mathrm{n}=422)\end{array}$ & $\begin{array}{l}\text { CTC-negative, } \\
\mathrm{n}(\%)(\mathrm{n}=1559)\end{array}$ & $p$-Value & $\begin{array}{c}\text { CA27.29-positive, } \\
\text { n }(\%)(\mathrm{n}=156)\end{array}$ & $\begin{array}{c}\text { CA27.29-negative, } \\
\text { n }(\%)(n=1825)\end{array}$ & $p$-Value \\
\hline Age (years) & & & $0.301^{\mathrm{a}}$ & & & $0.002^{\mathrm{a}}$ \\
\hline Median & 55 & 53 & & 57.5 & 53 & \\
\hline Range & $26-74$ & $21-78$ & & $28-75$ & $21-78$ & \\
\hline Tumor stage & & & $0.104^{b}$ & & & $0.012^{\mathrm{b}}$ \\
\hline pT1 & $158(37.4)$ & $665(42.7)$ & & $51(32.7)$ & $772(42.3)$ & \\
\hline pT2 & $229(54.3)$ & $795(51.0)$ & & $87(55.8)$ & $937(51.3)$ & \\
\hline pT3 & $29(6.9)$ & $72(4.6)$ & & $15(9.6)$ & $86(4.7)$ & \\
\hline pT4 & $6(1.4 \%)$ & $21(1.3)$ & & $3(1.9)$ & $24(1.3 \%)$ & \\
\hline Unknown & $0(0.0)$ & $6(0.4)$ & & $0(0.0)$ & $6(0.3)$ & \\
\hline Nodal stage & & & $<0.001^{\mathrm{b}}$ & & & $0.038^{\mathrm{b}}$ \\
\hline pNO & $129(30.6)$ & $533(34.2)$ & & $49(31.4)$ & $613(33.6)$ & \\
\hline $\mathrm{pN} 1$ & $176(41.7)$ & $741(47.5)$ & & $69(44.2)$ & $848(46.5)$ & \\
\hline $\mathrm{pN} 2$ & $68(16.1)$ & $206(13.2)$ & & $20(12.8)$ & $254(13.9)$ & \\
\hline $\mathrm{pN} 3$ & 48 (11.4) & $74(4.7)$ & & $18(11.5)$ & $104(5.7)$ & \\
\hline Unknown & $1(0.2)$ & $5(0.3)$ & & $0(0.0)$ & $6(0.3)$ & \\
\hline Histological grading & & & $0.242^{b}$ & & & $0.844^{\mathrm{b}}$ \\
\hline G1 & $14(3.3)$ & $82(5.3)$ & & $9(5.8)$ & $87(4.8)$ & \\
\hline $\mathrm{G} 2$ & $205(48.6)$ & $728(46.7)$ & & $74(47.4)$ & $859(47.1)$ & \\
\hline G3 & $202(47.9)$ & 746 (47.9) & & $73(46.8)$ & $875(47.9)$ & \\
\hline Unknown & $1(0.2)$ & $3(0.2)$ & & $0(0.0)$ & $4(0.2)$ & \\
\hline Histological type & & & $0.062^{b}$ & & & $0.001^{b}$ \\
\hline Ductal & $333(78.9)$ & $1267(81.3)$ & & $110(70.5)$ & $1490(81.6)$ & \\
\hline Lobular & $64(15.2)$ & $174(11.2)$ & & $33(21.2)$ & $205(11.2)$ & \\
\hline Other & $25(5.9)$ & $114(7.3)$ & & $13(8.3)$ & $126(6.9)$ & \\
\hline Unknown & $0(0.0)$ & $4(0.3)$ & & $0(0.0)$ & $4(0.2)$ & \\
\hline Estrogen receptor status & & & $0.547^{\mathrm{b}}$ & & & $0.637^{b}$ \\
\hline Negative & $121(28.7)$ & $470(30.1)$ & & $44(28.2)$ & $547(30.0)$ & \\
\hline Positive & $301(71.3)$ & $1087(69.7)$ & & $112(71.8)$ & $1276(69.9)$ & \\
\hline Unknown & $0(0.0)$ & $2(0.1)$ & & $0(0.0)$ & $2(0.2 \%)$ & \\
\hline Progesteron receptor status & & & $0.568^{b}$ & & & $0.941^{b}$ \\
\hline Negative & $140(33.2)$ & $539(34.6)$ & & $54(34.6)$ & $625(34.2)$ & \\
\hline Positive & $282(66.8)$ & $1016(65.2)$ & & $102(65.4)$ & $1196(65.5)$ & \\
\hline Unknown & $0(0.0)$ & $4(0.3)$ & & $0(0.0)$ & $4(0.2)$ & \\
\hline HER2 status & & & $0.792^{\mathrm{b}}$ & & & $0.663^{b}$ \\
\hline Negative & $314(74.4)$ & $1148(73.6)$ & & $118(75.6)$ & $1344(73.6)$ & \\
\hline Positive & $101(23.9)$ & $382(24.5)$ & & $36(23.1)$ & $447(24.5)$ & \\
\hline Unknown & $7(1.7)$ & $29(1.9)$ & & $2(1.3)$ & $34(1.9)$ & \\
\hline Menopausal status & & & $0.425^{\mathrm{b}}$ & & & $0.060^{\mathrm{b}}$ \\
\hline Premenopausal & $169(40.0)$ & $658(42.2)$ & & $54(34.6)$ & $773(42.4)$ & \\
\hline Postmenopausal & $253(60.0)$ & $901(57.8)$ & & $102(65.4)$ & $1052(57.6)$ & \\
\hline Type of surgery & & & $0.292^{\mathrm{b}}$ & & & $0.621^{b}$ \\
\hline Breast-conserving & $288(68.2)$ & $1103(70.8)$ & & 107 (68.6) & $1284(70.4)$ & \\
\hline Mastectomy & $134(31.8)$ & $453(29.1)$ & & $49(31.4)$ & $538(29.5)$ & \\
\hline Unknown & $0(0.0)$ & $3(0.2)$ & & $0(0.0)$ & $3(0.2)$ & \\
\hline
\end{tabular}

aMann-Whitney $U$-test, ${ }^{\text {b }}$ Chi-square test; tested without unknowns.

using frequency tables, and Pearson's chi-square tests were used in order to evaluate associations between marker positivity and categorical patient and tumor characteristics. Comparisons with regard to the non-normally distributed metric variable age were conducted with the Mann-Whitney $U$-test. The relationship between CTC positivity and CA27.29 positivity was assessed using Cramer's V, which is a measure of association between two nominal variables based on chi-square statistics. Cramer's V varies from 0 (no association between the variables) to 1 (complete association). $p$-Values were calculated two-sided and considered to be significant if they were below 0.05 . No adjustment for multiple testing was performed. The software IBM $^{\circledR}$ SPSS $^{\circledR}$ Statistics Version 21.0 (IBM Corp., Armonk, NY, USA) was used to perform the statistical analyses. 
Table II. Association between circulating tumor cells (positive if $\geq 1$ CTC/15 ml blood) and CA27.29 status (positive if $>31$ U/ml; negative if $\leq 31$ $\mathrm{U} / \mathrm{ml}$ ) at different times relative to administration of chemotherapy $(\mathrm{CHT})$.

\begin{tabular}{|c|c|c|c|c|}
\hline \multirow[b]{2}{*}{ Time point relative to $\mathrm{CHT}$} & \multirow[b]{2}{*}{ CTC status } & \multicolumn{2}{|c|}{ CA27.29 status } & \multirow[b]{2}{*}{ Total, n (\%) } \\
\hline & & Negative, n (\%) & Positive, n (\%) & \\
\hline \multirow[t]{3}{*}{ Before } & Negative, n (\%) & $1450(73.2)$ & $109(5.5)$ & $1559(78.7)$ \\
\hline & Positive, n (\%) & $375(18.9)$ & $47(2.4)$ & $422(21.3)$ \\
\hline & Total, n $(\%)$ & $1825(92.1)$ & $156(7.9)$ & $1981(100)^{\mathrm{a}}$ \\
\hline \multirow[t]{3}{*}{ Immediately after } & Negative, n (\%) & $968(60.4)$ & $269(16.8)$ & $1237(77.2)$ \\
\hline & Positive, n (\%) & $298(18.6)$ & $67(4.2)$ & $365(22.8)$ \\
\hline & Total, n (\%) & $1266(79.0)$ & $336(21.0)$ & $1602(100)^{\mathrm{b}}$ \\
\hline \multirow[t]{3}{*}{2 Years after } & Negative, n (\%) & $919(79.3)$ & $24(2.1)$ & $943(81.4)$ \\
\hline & Positive, $\mathrm{n}(\%)$ & $208(17.9)$ & $8(0.7)$ & $216(18.6)$ \\
\hline & Total, n $(\%)$ & $1127(97.2)$ & $32(2.8)$ & $1159(100)^{\mathrm{c}}$ \\
\hline \multirow[t]{3}{*}{5 Years after } & Negative, n (\%) & $607(85.9)$ & $40(5.7)$ & $647(91.5)$ \\
\hline & Positive, $\mathrm{n}(\%)$ & $47(6.6)$ & $13(1.8)$ & $60(8.5)$ \\
\hline & Total, n $(\%)$ & $654(92.5)$ & $53(7.5)$ & $707(100)^{\mathrm{d}}$ \\
\hline
\end{tabular}

$\mathrm{a} p=0.005$, Cramer's V $=0.063 ; \mathrm{b} p=0.162$, Cramer's V $=0.035 ;{ }^{\mathrm{c}} p=0.349$, Cramer's V $=0.028 ;{ }^{\mathrm{d}} p<0.001$, Cramer's V=0.164. $p$-Values were derived from chi-square test.

\section{Results}

CA27.29 and CTC data were available for 1,981 patients before and 1,602 patients immediately after CHT. Follow-up data for both CA27.29 and CTC were available for 1,159 patients at 2 years and for 707 patients at 5 years after completion of CHT.

Patient data. The association between patients' tumor characteristics and the presence of CTCs, as well as elevated CA27.29 levels (i.e. levels above $31 \mathrm{U} / \mu \mathrm{l}$ ) are presented in Table I. An elevated CA27.29 level was significantly related to tumor histology, age, tumor stage and nodal stage. We observed that $21.2 \%$ of all CA27.29-positive patients had lobular tumor histology compared to $11.2 \%$ of CA27.29negative patients $(p=0.001)$. The median age of CA27.29positive patients was 57.5 compared to 53.0 for CA27.29negative patients $(p=0.002)$. CA27.29-positive patients had also a higher proportion of pT2, pT3 and pT4 tumors $(p=0.012)$, as well as a higher proportion of $\mathrm{pN} 3$ tumors ( $p=0.038)$, compared to CA27.29-negative patients. Elevated CA27.29 level was not associated with grading, hormone receptor status, Receptor tyrosine-protein kinase ERBB-2 (HER2/neu) status, menopausal status or type of surgery. The presence of CTCs was only significantly associated with positive lymph node status; $27.5 \%$ of CTC-positive patients had $\mathrm{pN}$ of 2 or more compared to $17.9 \%$ of CTC-negative patients $(p<0.001)$.

CTC and CA27.29 levels before CHT and during follow-up. Before CHT, 156 patients (7.9\%) were CA27.29-positive and $422(21.3 \%)$ were CTC-positive. A total of 47 patients
(2.4\%) were simultaneously CA27.29- and CTC-positive and 484 patients $(24.4 \%)$ showed a discordant pattern of CA27.29 and CTC positivity (Table II). Pearson's chi-square test showed a significant but weak association between these two markers at this time point $(p=0.0015$, Cramer's $\mathrm{V}=0.063$ ).

Immediately after CHT, 336 patients (21.0\%) were CA27.29-positive and 365 (22.8\%) were CTC-positive. A total of 67 (4.2\%) were simultaneously CA27.29- and CTCpositive and 567 patients $(35.4 \%)$ were discordant in this regard (Table II); the association between CA27.29 and CTC was not significant at this time point $(p=0.162$; Cramer's $\mathrm{V}=0.035$ ).

Two years after CHT, 32 patients (2.8\%) were CA27.29positive and $216(18.6 \%)$ were CTC-positive. While eight patients $(0.7 \%)$ were simultaneously CA27.29- and CTCpositive, 232 patients $(20.0 \%)$ showed a discordant result in this regard, and the association between these two markers was not significant ( $p=0.349$; Cramer's V=0.028; Table II).

Five years after CHT, 53 patients $(7.5 \%)$ were CA27.29positive and $60(8.5 \%)$ were CTC-positive. 13 patients (1.8\%) were simultaneously CA27.29- and CTC-positive, while 87 patients $(12.3 \%)$ showed a discordant pattern in this regard. The association was significant but weak $(p=0.0015$; Cramer's V=0.164; Table II).

\section{Discussion}

Unfortunately no single prognostic marker exists which can reliably identify those $30 \%$ of patients with early-stage breast cancer who will experience relapse during follow-up. In this 
regard, CA27.29 and CTC are no exception, as in our study positivity rates for both parameters were considerably lower than $30 \%$ at any of the examined time points.

CTCs. We found $21.3 \%$ of the patients to be CTC-positive before start of CHT and $22.8 \%$ thereafter. This is well in line with reports from other studies. Lucci et al. reported a positivity rate of $24 \%$ in a group of 302 patients using the same system in the same setting (5). The neoadjuvant trials GeparQuattro and Remagus 02 reported comparable positivity rates before the start of neoadjuvant treatment (22\% and 23\%) (14-16). Mikulová et al. reported 31\% prevalence of CTCs in adjuvant/neoadjuvant breast cancer patients before start of CHT using the AdnaTest Breast Cancer ${ }^{\mathrm{TM}}$ (17). If only the adjuvant cohort is considered, the CTC positivity rate was $22 \%$ and thus almost identical to the rate found in our study. Of note, they also reported on CTC positivity after completion of $\mathrm{CHT}$ and found 7\% CTC-positive patients, which is considerably lower than the $22.8 \%$ found in our study (17). Given the small number of patients and the different CHT regimen used in that study, it is difficult to evaluate whether this observed difference is attributable to the test method.

During follow-up, we tested for CTCs at 2 and 5 years and found $18.6 \%$ and $8.5 \%$ CTC-positive, relapse-free patients respectively. To our knowledge, no other study reported on prevalence rates of CTC in relapse-free patients after such follow-up periods in the adjuvant setting. Nevertheless there are data about dormant tumor cells in long-term relapse-free survivors, reporting CTCs in 36\% of patients at 8 to 22 years after mastectomy for early breast cancer (18). Therefore, our data emphasize the concept of tumor dormancy in relapse-free patients, meaning that a significant proportion of patients with detectable CTCs during follow-up will not face relapse.

CA27.29. Prevalence of CA27.29 before CHT within the SUCCESS study has been reported and discussed elsewhere (12). Rising from $7.9 \%$ to $21.0 \%$, we see a distinct increase after CHT. To our knowledge, there is no report on CA27.29 and its course during follow-up after CHT in early-stage breast cancer. Nevertheless, Kiang et al. described a similar increase for CA15-3 and carcinoembryonic antigen in patients with advanced breast cancer as 'flare up' effect due to cytolysis and subsequent increased release of MUC1 into the bloodstream (19). Furthermore, this therapy-related surge has been described for various tumor entities and different tumor markers, and raises the question about possible prognostic or therapeutic relevance (20). It therefore seems reasonable to evaluate the prognostic value of CA27.29 at this time point separately.

A total of $2.8 \%$ and $7.8 \%$ relapse-free patients had an elevated CA27.29 level after 2 and 5 years of follow-up, respectively. Since comparable data are lacking, we can only speculate that CA 27-29 represents a prognostic marker for late relapse. Long-term follow-up investigation will be needed to verify this assumption.

Association of CTC positivity and CA27.29. Associating CTCs with CA27.29, our study shows a weak, but significant association before and 5 years after CHT. No association was found to exist directly after CHT and after 2 years of followup. A possible explanation for the overall weak association could be that both markers are known to represent different aspects of the disease and therefore a different reaction of the two markers to therapy seems reasonable. As shown in Table I, the association of CA27.29 with other known prognostic markers is strongest with tumor size. This is consistent with the aforementioned representation of total tumor mass by CA27.29 $(11,12)$. In contrast to MUC1 tumor markers such as CA27.29, CTC positivity seems to indicate more the potential for dissemination itself rather than only reflecting tumor mass $(21,22)$. In line with this, CTC positivity correlated best with nodal status in our study, interpretable as an early indication of a high potential for dissemination (2). This is consistent with data from the metastatic setting which showed only a weak association between CA15-3 level and CTC count during therapy (22).

The fact that both markers seem to represent different aspects of the disease and the fact that their association - if any - throughout follow-up is rather weak makes the evaluation of their potentially independent prognostic value very interestingly. As for other tumor entities, such as gall bladder carcinoma, the combined use of different tumor markers may lead to a more precise assessment of prognosis (23).

Study limitations. Unfortunately not all initially CTC- or CA27.29-positive patients were available for analysis up to 5 years of follow-up and patients with relapsing disease were excluded from further analysis.

\section{Conclusion}

It is imperative to identify those patients at highest risk for recurrence in order to develop tailored treatment strategies and thereby prevent relapse. In this regard, this study adds valuable data by presenting for the first time data on prevalence, course and association of two promising markers, CA27.29 and CTC positivity, in an adjuvant setting during a follow-up period of 5 years. We showed that positivity for CTCs and CA27.29 were significantly but only weakly associated before $\mathrm{CHT}$ and 5 years after CHT, while no association was found immediately and 2 years after CHT during the course of early-stage breast cancer. The lack of a strong association between the two markers suggests that they might provide valuable independent prognostic information. 
Two main questions emerge from this study and should be addressed in the future. Firstly, as stated before, the prognostic value of the marker combination needs to be assessed in order to evaluate whether the two markers indeed have independent prognostic relevance. Secondly, it remains to be shown whether patients that are positive for both CA27.29 and CTCs at any one time represent a high-risk subgroup that might need a tailored or more intense treatment regimen.

\section{References}

1 World Cancer Report. Stewart BW and Wild CP (eds.). Lyon, WHO/IARC, 2014.

2 Braun S and Naume B: Circulating and disseminated tumor cells. J Clin Oncol 23: 1623-1626, 2005.

3 Braun S, Vogl FD, Naume B, Janni W, Osborne MP, Coombes RC, Schlimok G, Diel IJ, Gerber B, Gebauer G, Pierga J-Y, Marth C, Oruzio D, Wiedswang G, Solomayer E-F, Kundt G, Strobl B, Fehm T, Wong GYC, Bliss J, Vincent-Salomon A and Pantel K: A pooled analysis of bone marrow micrometastasis in breast cancer. N Engl J Med 353: 793-802, 2005.

4 Rack B, Schindlbeck C, Jückstock J, Andergassen U, Hepp P, Zwingers T, Friedl TWP, Lorenz R, Tesch H, Fasching P a, Fehm T, Schneeweiss A, Lichtenegger W, Beckmann MW, Friese K, Pantel K and Janni W: Circulating tumor cells predict survival in early average-to-high risk breast cancer patients. J Natl Cancer Inst 106: 1-11, 2014.

5 Lucci A, Hall CS, Lodhi AK, Bhattacharyya A, Anderson AE, Xiao L, Bedrosian I, Kuerer $\mathrm{HM}$ and Krishnamurthy S: Circulating tumour cells in non-metastatic breast cancer: a prospective study. Lancet Oncol 13: 688-695, 2012.

6 Harris L, Fritsche H, Mennel R, Norton L, Ravdin P, Taube S, Somerfield MR, Hayes DF, Bast RC Jr. RCB and Arbor A: American Society of Clinical Oncology 2007 Update of Recommendations for the Use of Tumor Markers in Breast Cancer The American Society of Clinical Oncology. J Clin Oncol 25: 5287-5312, 2008.

7 Martín A, Corte MD, Alvarez AM, Rodriguez JC, Andicoechea A, Bongera M, Junquera S, Pidal D, Allende T, Muñiz JLG and Vizoso F: Prognostic value of pre-operative serum CA 15.3 levels in breast cancer. Anticancer Res 26: 3965-3971, 2006.

8 Ebeling F, Stieber P, Untch M, Nagel D, Konecny G, Schmitt U, Fateh-Moghadam A and Seidel D: Serum CEA and CA15-3 as prognostic factors in primary breast cancer. $\mathrm{Br} \mathrm{J}$ Cancer 86: 1217-1222, 2002.

9 Gion M, Boracchi P, Dittadi R, Biganzoli E, Peloso L, Mione R, Gatti C, Paccagnella A and Marubini E: Prognostic role of serum CA15.3 in 362 node-negative breast cancers. An old player for a new game. Eur J Cancer 38: 1181-1188, 2002.

10 Kumpulainen EJ, Keskikuru RJ and Johansson RT: Serum tumor marker CA15.3 and stage are the two most powerful predictors of survival in primary breast cancer. Breast Cancer Res Treat 76: 95-102, 2002.

11 Croce M V, Isla-Larrain MT, Demichelis SO, Gori JR, Price MR and Segal-Eiras A: Tissue and serum MUC1 mucin detection in breast cancer patients. Breast Cancer Res Treat 81: 195-207, 2003.
12 Rack B, Schindlbeck C, Jückstock J, Genss E-M, Hepp P, Lorenz R, Tesch H, Schneeweiss A, Beckmann MW, Lichtenegger W, Sommer H, Friese K and Janni W: Prevalence of CA27.29 in primary breast cancer patients before the start of systemic treatment. Anticancer Res 30: 1837-1841, 2010.

13 Cristofanilli M: Circulating tumor cells, disease progression, and survival in metastatic breast cancer. Semin Oncol 33: S9-14, 2006.

14 Bidard F-C, Mathiot C, Delaloge S, Brain E, Giachetti S, de Cremoux P, Marty M and Pierga J-Y: Single circulating tumor cell detection and overall survival in nonmetastatic breast cancer. Ann Oncol 21: 729-733, 2010.

15 Mueller V, Riethdorf S, Loibl S, Komor M, Houber J, Schrader I, Conrad U, Untch M, vMinckwitz G and Pantel K: Prospective monitoring of circulating tumor cells in breast cancer patients treated with primary systemic therapy--A translational project of the German Breast Group study GeparQuattro. ASCO Meet Abstr 25: 21085, 2007.

16 Pierga J-Y, Bidard F-C, Mathiot C, Brain E, Delaloge S, Giachetti S, de Cremoux P, Salmon R, Vincent-Salomon A and Marty M: Circulating tumor cell detection predicts early metastatic relapse after neoadjuvant chemotherapy in large operable and locally advanced breast cancer in a phase II randomized trial. Clin Cancer Res 14: 7004-7010, 2008.

17 Mikulová V, Cabiňaková M, Janatková I, Mestek O, Zima T and Tesařová $\mathrm{P}$ : Detection of circulating tumor cells during followup of patients with early breast cancer: Clinical utility for monitoring of therapy efficacy. Scand J Clin Lab Invest 74: 132142, 2014.

18 Meng S, Tripathy D, Frenkel EP, Shete S, Naftalis EZ, Huth JF, Beitsch PD, Leitch M, Hoover S, Euhus D, Haley B, Morrison L, Fleming TP, Herlyn D, Terstappen LWMM, Fehm T, Tucker TF, Lane N, Wang J and Uhr JW: Circulating tumor cells in patients with breast cancer dormancy 8152-8162, 2004.

19 Kiang DT, Greenberg LJ and Kennedy BJ: Tumor marker kinetics in the monitoring of breast cancer. Cancer 65: 193-199, 1990.

20 Mundle SD, Marathe AS and Chelladurai M: Transient therapyrelated surge in serum tumor biomarkers: characterizing behavior and postulating its biologic role. Crit Rev Oncol Hematol 86: 15-22, 2013.

21 Cristofanilli M, Budd GT, Ellis MJ, Stopeck A, Matera J, Miller MC, Reuben JM, Doyle G V, Allard WJ, Terstappen LWMM and Hayes DF: Circulating tumor cells, disease progression, and survival in metastatic breast cancer. N Engl J Med 351: 781-791, 2004.

22 Hartkopf AD, Wagner P, Wallwiener D, Fehm T and Rothmund $\mathrm{R}$ : Changing Levels of Circulating Tumor Cells in Monitoring Chemotherapy Response in Patients with Metastatic Breast Cancer 984: 979-984, 2011.

23 Wang Y-F, Feng F-L, Zhao X-H, Ye Z-X, Zeng H-P, Li Z, Jiang $\mathrm{X}-\mathrm{Q}$ and Peng Z-H: Combined detection tumor markers for diagnosis and prognosis of gallbladder cancer. World $\mathbf{J}$ Gastroenterol 20: 4085-4092, 2014. 